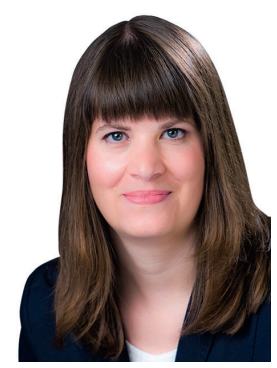

Stephanie Joachim

Koordination «OphthalmoCampus»

\title{
«Unser Fach mit Zukunft»
}

In dieser Ausgabe nutzen wir die Gelegenheit, den Kongress der Deutschen Ophthalmologischen Gesellschaft, der Ende September in Berlin stattfand, noch einmal Revue passieren zu lassen. Bei 1200 wissenschaftlichen Beiträgen und 281 Sitzungen ist es natürlich schwierig, dem Kongress gerecht zu werden, die von uns ausgewählten Artikel geben aber einen Eindruck über «unser Fach mit Zukunft».

Zukunftsweisend ist auch die Zulassung der ersten okulären Gentherapie. Prof. Dr. Dr. Dominik Fischer, der maßgeblich an der Entwicklung dieser Therapie beteiligt war, erläutert uns im Interview die Möglichkeiten und Perspektiven dieser neuen Therapieform.

\section{Kongresse}

DOG 2019: «Wir können die Zukunft nur mit Forschung gestalten»

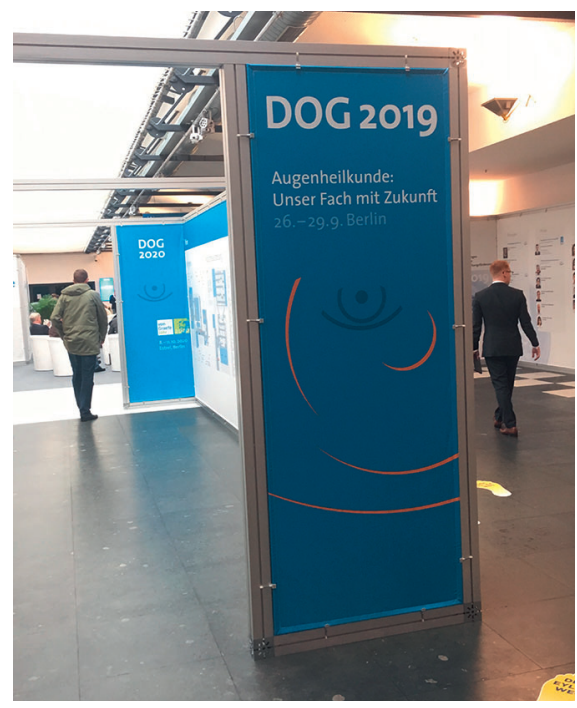

Die DOG 2019 fand vom 26. bis 29. September in Berlin statt.

Künstliche Intelligenz (KI), Daten-Register und der verstärkte Austausch zwischen klinisch tätigen Augenärzten und Grundlagenwissenschaftlern werden die Betreuung augenärztlicher Patienten weiter verbessern. Das ist ein zentrales Ergebnis der DOG
2019, die unter dem Motto «Augenheilkunde: Unser Fach mit Zukunft» am Sonntag, den 29. September, in Berlin endete. Mehr als 5100 Teilnehmer kamen, davon 3169 Fachteilnehmer - eine Rekordbeteiligung in der 162-jährigen Geschichte der ältesten augenfachärztlichen Gesellschaft der Welt. Zu den Highlights zählten neben den Keynote Lectures die neuen Sitzungsformate «Highlights in Translational Science» und «DOG International Experts Day».

«Highlights in Translational Science» brachte klinische Forscher und Grundlagenwissenschaftler zusammen: Augenärzte erfuhren, in welchen Bereichen sich Laborforschung auf dem Weg ans Krankenbett befindet, umgekehrt sahen Naturwissenschaftler, welche Erkrankungen man wie behandeln kann und wo noch Verbesserungsbedarf besteht. «Das war ein sehr fruchtbarer Dialog», bilanziert Prof. Dr. Claus Cursiefen, Präsident der Deutschen Ophthalmologischen Gesellschaft (DOG). Der «DOG International Experts Day» wiederum förderte als ganztägiges englischsprachiges Programm den internationalen Austausch mit Top-Spezialisten. «Probleme, die sich in der Behandlung auftun, müssen wir mit translationaler Forschung angehen, sie sind eigentlich nur interdisziplinär und in internationaler Zusammenarbeit zu lösen», ist Cursiefen überzeugt. «Wir können Zukunft nur mit Forschung gestalten.»

\section{KARGER}

Fax +497614520714

information@karger.com

www.karger.com

\section{(c) 2019 S. Karger GmbH, Freiburg}


Im kommenden Jahr findet die DOG 2020 unter der Präsidentschaft von Prof. Dr. Hans Hoerauf vom 8. bis 11. Oktober 2020 in Berlin statt wie gewohnt im Estrel. Der Direktor der Universitäts-Augenklinik Göttingen wird das 150. Todesjahr von Albrecht von Graefe, des Begründers der DOG, in den Vordergrund rücken. Zudem wird der Kongress 2020 als Joint Meeting mit der International Society of Ocular Trauma (ISOT) abgehalten. Darüber hinaus findet auch das HerbstExamen des European Board of Opththalmology (EBO) erstmals im Rahmen des DOG-Kongresses statt. Zu den zentralen Kongressthemen werden neben Forschung, Innovation, Diagnostik und Therapie auch die Auswirkungen der Ökonomisierung auf die Medizin im Allgemeinen und die Augenheilkunde im Besonderen zählen.

\section{Augenuntersuchung per Smartphone erkennt Schädigung des Sehnervs und der Netzhaut}

In vielen Entwicklungs- und Schwellenländern sind finanzielle Ressourcen knapp und die Anzahl an Augenärzten unzureichend. Demgegenüber sind Smartphones mittlerweile weltweit verfügbar, mit guten Kameraeigenschaften und intuitiver Bedienung. Forscher der Universitäts-Augenklinik Bonn haben daher in Süd-Indien erprobt, ob eine Augenhintergrund-Untersuchung mit der Smartphone-Kamera die Erkennung von diabetesbedingter Retinopathie ermöglicht - mit Erfolg.

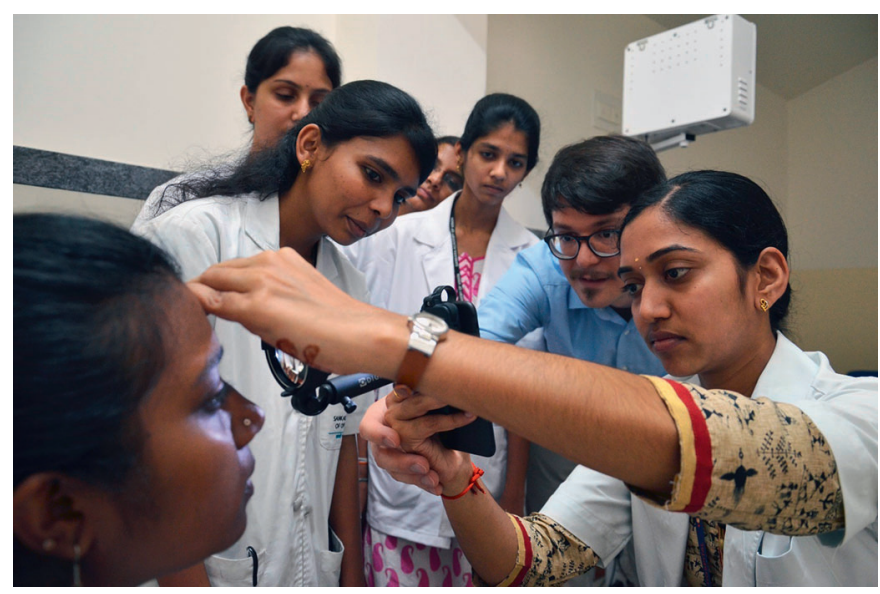

Schulung augenärztlichen Hilfspersonals am Sankara Eye Hospital, Bangalore, Indien durch Dr. Wintergerst von der Universitäts-Augenklinik Bonn.

Konkret nutzten die Wissenschaftler vor Ort Adapter, die vor die Smartphone-Kamera geschaltet werden. «In dieser Kombination können wir den Augenhintergrund auf diabetische Retinopathie untersuchen oder auf Schädigungen des Sehnervs, um etwa einen Grünen Star frühzeitig zu erkennen», erläutert Projektleiter Dr. Maximilian Wintergerst (Bonn). «Wir haben verschiedene Ansätze getestet und mit dieser Variante sehr gute Erfahrungen gemacht». Die Studie wird demnächst veröffentlicht.

Auch augenärztliches Assistenzpersonal kann die digitale FundusUntersuchung durchführen. «Das eröffnet die Chance auf einen telemedizinischen Ansatz», sagt Wintergerst. «Das Assistenzpersonal schickt die mit dem Smartphone aufgenommenen Fundus-Bilder per Internet an ein Krankenhaus, wo sie von Augenärzten am Computer beurteilt werden.»
Damit ist dieser Ansatz nicht nur für Länder mit mittlerer Einkommensstruktur wie Indien interessant. «Auch eine Anwendung in Deutschland wäre denkbar, etwa zur Untersuchung und Dokumentation bei bettlägerigen Patienten auf der Intensivstation oder bei Patienten in Altersheimen, die nicht ohne weiteres zum Augenarzt gehen können», meint der DOG-Experte.

\section{Augenärzte testen Kontaktlinsen mit UV-Schutz}

Limbale Stammzellen (LSC) sind für ein gesundes Sehen von entscheidender Bedeutung. Ohne diese Zellen würde die Hornhaut trübe und damit die Sehkraft eingeschränkt. UV-Licht, aber auch Krankheit, Verbrennungen oder Verletzungen durch Chemikalien können diese Stammzellen schädigen und zur Erblindung führen. «Inzwischen gibt es jedoch mehrere Verfahren, wie man limbale Stammzellen transplantieren und damit die Augenoberfläche wieder herstellen kann», berichtet Prof. Dr. Claus Cursiefen (Köln). Nach der Transplantation sind diese Stammzellen jedoch sehr empfindlich gegenüber UV-Strahlen.

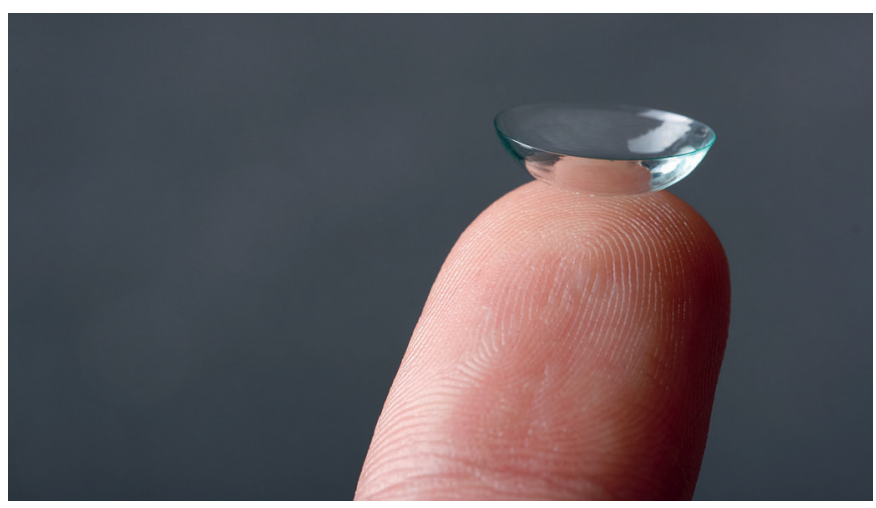

Kontaktlinsen mit UV-Schutz könnten zukünftig die Hornhaut ihrer Träger vor Schäden durch UV-Strahlung schützen.

Um Schäden abzuwenden und das Sehvermögen der Patienten zu erhalten, testeten die Kölner Augenärzte auf dem Markt verfügbare Kontaktlinsen mit eingebautem UV-Filter. «Wir führten dafür Versuche an humanen limbalen Stammzellen durch, die im Labor mit UV-Licht bestrahlt wurden», berichtet Cursiefen. Ergebnis: «Die Kontaktlinsen mit eingebautem Filter konnten UV-Schäden fast vollständig verhindern, negative Effekte blieben aus». Ohne UVSchutz traten an den limbalen Stammzellen deutliche Schäden ein. Jetzt bereiten die Kölner Wissenschaftler eine klinische Studie vor: Patienten, die eine limbale Stammzell-Transplantation erhalten haben, sollen nach der Operation Kontaktlinsen mit UV-Schutz tragen. «Sollte der UV-Filter in den Kontaktlinsen bei diesen Patienten funktionieren, wären die Haftschalen auch eine Option für Menschen, die viel Zeit draußen im Tageslicht verbringen», meint Cursiefen.

\section{Atropin-Augentropfen bremsen Kurzsichtigkeit}

Um Kurzsichtigkeit aufzuhalten, stehen verschiedene Ansätze bereit. «Täglich 2 Stunden Aufenthalt im Freien bei Tageslicht halbieren das Risiko für Kurzsichtigkeit», erläutert Prof. Dr. Wolf Lagrèze (Freiburg). «Längeres Lesen in einem Abstand von weniger als 30 


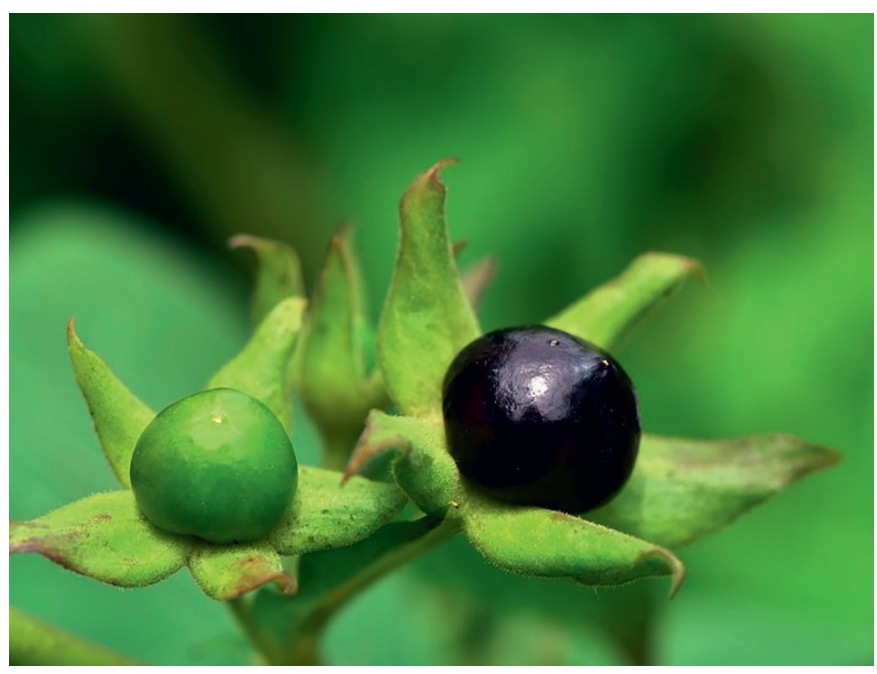

Wirksame und verträgliche Therapie mit Tollkirsche.

cm sollte vermieden werden», fügt der Freiburger Augenarzt hinzu. Darüber hinaus gibt es spezielle Kontaktlinsen, die das Fortschreiten der Kurzsichtigkeit um bis zu 40\% mindern können.

Am wirksamsten hat sich jedoch eine Therapie mit Atropin-Augentropfen erwiesen. Dass Atropin, eine Substanz aus der Tollkirsche, Kurzsichtigkeit aufhalten kann, ist seit mehr als 100 Jahren bekannt. «Wegen ihrer Nebenwirkungen - Blendung und Nahsichtstörung - wurden Atropin-Tropfen zu diesem Zweck aber kaum verordnet», berichtet Lagrèze. Das hat sich jetzt geändert. Denn Forscher aus Singapur haben eine Konzentration gefunden, die das Fortschreiten der Kurzsichtigkeit um bis zu 50\% mindert und gleichzeitig weitgehend nebenwirkungsfrei ist.

Dass Atropin-Tropfen in der geringen Konzentration von 0,01\% gut wirken und dabei verträglich sind, belegen inzwischen große und aussagekräftige Studien aus Asien. «Seit der Veröffentlichung dieser Daten hat sich die Anwendung niedrig dosierter Atropin-Augentropfen weltweit sehr schnell durchgesetzt und wird auch in Deutschland seit wenigen Jahren von vielen Augenärzten in Kliniken und Praxen eingesetzt», betont Lagrèze. So haben verschiedene Länder, darunter Deutschland, Leitlinien und Behandlungsempfehlungen formuliert.

Für die Atropin-Therapie kommen Kinder im Alter von 6 bis 14 Jahren in Frage, bei denen die Kurzsichtigkeit pro Jahr um mindestens eine halbe Dioptrie zunimmt. «Die Eltern geben abends vor dem Zubettgehen jeweils einen Tropfen in jedes Auge», erklärt Lagrèze. «Wichtig ist eine Tropfen-Zubereitung ohne Konservierungsmittel», betont Lagrèze. Zudem muss der Augenarzt die Eltern darauf hinweisen, dass es sich bei dieser Behandlung um einen sogenannten Off-Label-Use handelt. «Nach 2 Jahren Therapiedauer entscheidet der Augenarzt, ob die Behandlung fortgesetzt werden sollte».

Kontaktadresse

Pressestelle DOG, Kerstin Ullrich, Postfach 3011 20, 70451 Stuttgart, Deutschland, ullrich@medizinkommunikation.org 


\section{Experte im Gespräch}

\section{«Je weniger ein Patient sieht, desto wertvoller ist der Rest an Sehfunktion» - die erste okuläre Genthera- pie bei erblicher Netzhautdegeneration}

In der Ausgabe 2/17 des KARGER KOMPASS OPHTHALMOLOGIE hat Prof. Dr. Dr. Dominik Fischer das erste Mal über die Funktionsweisen und Möglichkeiten der Gentherapie in der Augenheilkunde berichtet. Eine der damals laufenden Studien untersuchte eine Gentherapie, die eine Behandlung einer Netzhautdystrophie aufgrund einer biallelischen Mutation auf dem RPE65-Gen versprach. Diese Mutation bewirkt, dass das von dem Gen kodierte Enzym RPE65 nicht, nur in geringen Mengen oder nur mit verminderter Funktionalität von den Zellen hergestellt werden kann, was wiederum zu einer verminderten oder nicht vorhandenen Aktivität des Sehzyklus führt. Auf diesen Zyklus sind die Stäbchen zu 100\% angewiesen, weswegen ein Funktionsverlust des Enzyms RPE65 vor allem diese betrifft. Bei den Patienten macht sich dies bemerkbar durch eine frühe Nachtblindheit. Die Zapfen werden zum gewissen Teil von anderen Quellen versorgt, weswegen hier häufig in frühen Phasen noch ein gewisses Maß an Funktionalität vorliegt. Luxturna ${ }^{\oplus}$ (Voretigene neparvovec) erhielt im März 2018 die Zulassung durch die amerikanische Food and Drug Administration (FDA), die Zulassung durch die Europäische Arzneimittelbehörde (EMA) folgte im November des gleichen Jahres.

Auf dem Kongress der Deutschen Ophthalmologischen Gesellschaft hat sich Prof. Dr. Dr. Dominik Fischer Zeit für uns genommen, um uns zu berichten, was sich seit unserem letzten Gespräch getan hat und wo die Gentherapie in der Augenheilkunde heute steht. Das Interview führte unsere Redakteurin Julia Weber.

Prof. Dr. Dr. Dominik Fischer, das letzte Mal haben wir uns im Frühjahr 2017 unterhalten, damals war Luxturna ${ }^{\circledR}$ noch in der Studienphase. Was hat sich seitdem getan?

Seitdem hat sich sehr viel getan - primär natürlich die Zulassung von Luxturna als Medikament, zunächst durch die Food and Drug Administration (FDA) und dann durch die Europäische Arzneimittelbehörde (EMA). Das allein war an sich schon ein ganz spannender Prozess, bei dem ich als Gutachter dabei war und selbst erfahren durfte, wie Patientenvertreter gegenüber der EMA argumentiert haben. Zum Beispiel zu Fragen wie: «Wie fortgeschritten darf die Erkrankung sein bzw. wie schlecht darf jemand sehen, damit er noch von der Therapie profitieren darf?»In anderen Worten: Sollte man einen älteren Menschen, bei dem die Netzhautdegeneration schon sehr weit fortgeschritten ist, noch therapieren? Ist es das noch wert?

Eine Patientenvertreterin antwortete darauf: «Je weniger ein Patient sieht, desto wertvoller ist der Rest an Sehfunktion.» Und das ist ein sehr schlagfertiges Argument, das die Behörden dann auch da- von überzeugt hat, keine Obergrenze auszugeben, sondern es den behandelnden Ärzten zu überlassen festzustellen, ob noch genug Netzhautgewebe da ist, damit die Patienten von der Therapie profitieren können.

Das bedeutet, es gibt es keine harte Linie, ab der man sagt: Okay, ab hier nicht mehr?

Exakt, im Moment ist es noch eine individuelle Fallabschätzung, ob die Therapie für den Patienten die richtige ist und ob der Patient geeignet ist. Zusätzlich muss man abschätzen, welche Prognose man dem Patienten geben kann. Das ist ein spannender Prozess, der aber natürlich auch die Ungewissheiten ein Stück weit auf die behandelnden Ärzte überträgt. Diese Entscheidung ist ja nicht unerheblich. Sie ist für den Patienten nicht unerheblich, der sich einer Operation unterzieht, und sie ist für die Klinik und natürlich den Kostenträger kein unerheblicher Aufwand. Das muss man abwägen.

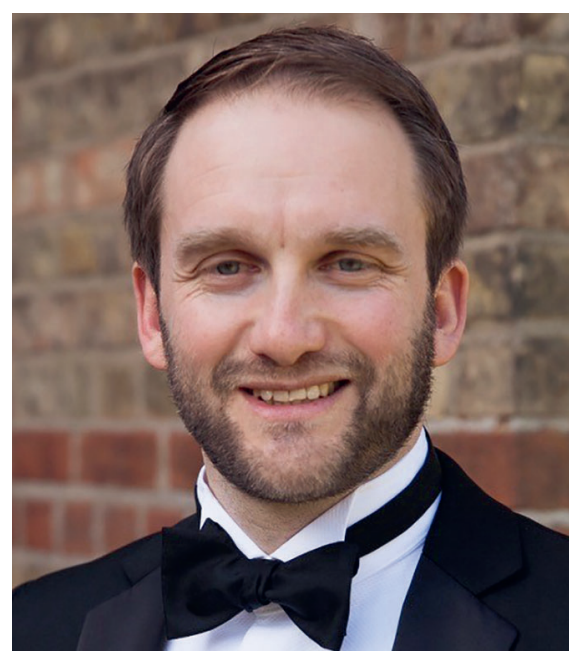

«Es war ein besonderes Erlebnis, als ich einem jungen Mann gegenübersaß, der im Wesentlichen das Produkt bekommen hat, das ich damals als PhD-Student entwickelt hatte.»
Wer trifft bisher diese Entscheidungen? Im Moment ist es so, dass die Patienten in erster Linie aus unserer «eigenen Kartei» kommen. Wir kennen einen Großteil von ihnen schon über viele Jahre und möchten sie jetzt natürlich gerne einer Therapie zuführen, solange wir davon überzeugt sind, dass sie davon einen Vorteil haben. In Zukunft wird es natürlich so sein, dass die Patienten vom niedergelassenen Kollegen überwiesen werden.

Wie sieht das konkret aus? Sie hatten vorhin schon einmal die Kostenträger erwähnt.

Konkret für Deutschland heißt das: Wir stellen Einzelfallanträge für Patienten, bei denen wir es für sinnvoll erachten, die Therapie durchzuführen. Dieser Einzelfallantrag geht an die Krankenkasse des Patienten, welche dann in der Regel ein Gutachten durch ihren medizinischen Dienst einholt. Oftmals sind das allerdings Kollegen, die keine Augenärzte sind und keine spezielle Kenntnis von erblichen Netzhauterkrankungen, geschweige denn von Gentherapie, haben. Das heißt man argumentiert da mit Leuten, die naturgemäß weniger von der Sachfrage verstehen als man selber, was mitunter etwas frustrierend sein kann. Nichtsdestotrotz kann man natürlich nachvollziehen, dass die Krankenkasse auch eine externe Einschätzung haben möchte. Sobald die Krankenkasse dann eine Zusage zur Kostenübernahme schreibt, können wir den nächsten Schritt gehen und die tatsächliche Behandlung planen. 
Können Sie sagen, wie viele Patienten ungefähr schon behandelt wurden?

In Deutschland wurden meiner Kenntnis nach 2 Patienten behandelt, in Europa 12 und weltweit knapp 100.

Gibt es schon erste Erkenntnisse, wie lange der Effekt der Gentherapie anhält?

Sehr eindrücklich war neulich auf dem Kongress der European Society of Retina Specialists (EURETINA, 05.-08. September 2019, Anm.d.Red.) in Paris die Vorstellung des Patienten, der als erster überhaupt Luxturna im Rahmen der Phase-I-Studie bekommen hat. Damals ein kleiner Junge, heute ist er 22 Jahre alt, ein eigenständiger, selbstbewusster junger Mann und studiert Psychologie. Dieser Patient hat auf dem Podium erzählt, wie sich sein Leben geändert hat und wie es seitdem stabil besser geworden ist. Man kann festhalten, dass die Therapiewirkung zumindest in einzelnen Fällen seit vielen Jahren anhält und zu einer deutlichen und individuell stark wahrnehmbaren Verbesserung der Lebensqualität geführt hat.

Ohne Therapie wäre er wahrscheinlich aufgrund der Netzhautdegeneration erblindet?

Davon kann man ausgehen. Der Verlauf ist individuell ein bisschen unterschiedlich. So gibt es Leute, die aufgrund der Mutationen schon von Kind auf blind sind. Es gibt Personen, die eine Mutation mit sich tragen, aber bis zum Erwachsenenalter noch so gut sehen, dass sie nicht blind im Sinne des Gesetzes sind. Im konkreten Fall hat der Patient berichtet, dass es eine Sehfunktionsverbesserung bei ihm gab, die seit der Behandlung angehalten hat. Das macht natürlich Mut, muss aber nicht heißen, dass es bei jedem Patienten so gut funktioniert. Es zeigt aber das Potenzial der Therapie auf.

Sie sind vorhin darauf eingegangen, dass die Entscheidung, ob eine Therapie sinnvoll ist oder nicht, individuell getroffen werden muss. Was wären denn Voraussetzungen, damit sich eine Therapie lohnt?

Es gibt zunächst 3 Kriterien, die erfüllt sein müssen: Das wichtigste Kriterium ist die klinische Diagnose, also dass der Patient offensichtlich eine Netzhautdegeneration hat. Das zweite ist die genetische Diagnose, die bestätigt, dass auf beiden Allelen eine Mutation in dem RP65-Gen vorliegt. Und das dritte Kriterium ist, dass noch genug Netzhautgewebe da ist, d.h. genügend Zielzellen, in die das Gen aus dem Wirkstoff dann reingehen kann.

Gibt es Voraussetzungen, die der Operateur mitbringen muss, um den Eingriff vornehmen zu können?

Ja, die gibt es. Das sind zunächst formalen Kriterien: Den Eingriff kann nur jemand durchführen, der Erfahrung mit vitreoretinaler Chirurgie hat. Zusätzlich muss er ein Training absolvieren, das von Novartis angeboten wird, und sich in den spezifischen Aspekten der subretinalen Gentherapie weiterbilden. Darüber hinaus gibt es natürlich inhaltlich Voraussetzungen, die wichtig sind.
Welches sind diese inhaltlichen Voraussetzungen?

Konkret braucht man ein Team, das verschiedene Aspekte abdecken kann: Im Team muss jemand sein, der Erfahrung hat mit Patienten mit erblichen Netzhauterkrankungen, der auch sicher die klinische Diagnose stellen kann. Es muss im Team jemand sein, der die molekulargenetische Diagnostik versteht und bestätigen kann, dass die genetische Diagnose mit der klinischen Diagnose übereinstimmt. Das ist nicht trivial. Und dann muss jemand dabei sein, der die vitreoretinal-chirurgischen Aspekte abdecken kann - gerade, wenn es sich um junge Patienten handelt.

\section{Sie haben erwähnt, dass es im Zuge der Zulassung vom Alter der Patienten her keine Beschränkung nach oben gibt, aber nach unten. Wann ist der früheste Punkt, ab dem therapiert werden kann?}

Im Wesentlichen gibt es darauf 2 Antworten, eine von der FDA und eine von der EMA: Die FDA hat aufgrund theoretischer Überlegungen beschlossen, dass die Therapie nicht bei Kindern unter $12 \mathrm{Mo-}$ naten durchgeführt werden soll, da sich das Zielgewebe, das retinale Pigmentepithel, bis zu diesem Alter noch teilt. Das heißt, wenn man früher operieren würde, würde man beispielsweise 5 Zellen therapieren, die sich dann in 10 Zellen teilen, wovon dann die Hälfte gar nicht therapiert wäre.

\section{Und was sagt die EMA?}

Die EMA hat nach Diskussionen mit verschiedenen Experten beschlossen, dass wir zu wenig wissen, um beurteilen zu können, ab welchem Alter die Therapie frühestens sinnvoll ist. Was wir jedoch kennen, sind die Daten aus der Zulassungsstudie, in der die jüngsten Patienten 4 Jahre alt waren. Das hat sich dann niedergeschlagen in der Verlautbarung: «Es gibt keine Erfahrung über die Sicherheit und Wirksamkeit der Behandlung bei Patienten unter 4 Jahren.» Das heißt nicht, dass man als Arzt keine Patienten, die jünger als 4 Jahre sind, therapieren darf. Es heißt bloß, dass wir bisher damit keine Erfahrung haben. Gerade Chirurgen mit Erfahrung in der Kinderchirurgie haben eine gewisse Scheu, so einen Eingriff bei Säuglingen durchzuführen: Die Anatomie ändert sich, das Gewebe ändert sich, und wahrscheinlich ist das OP-Risiko höher. Das wichtigste Kriterium für uns Ärzte ist immer das oberste Prinzip «nihil non nocere»: keinen Schaden anrichten. Und da will man natürlich ungerne einen 2-jährigen operieren und dann hinterher mehr schaden als nutzen. Es kann sein, dass man mit der Zeit merkt, dass man dem Patienten zu mehr Vorteil verhilft, je früher man die Therapie anwendet. Aber bis jetzt fehlen uns die Erfahrungswerte dazu.

\section{Wie geht es jetzt mit der Gentherapie selbst weiter? Gibt es weitere Beobachtungsstudien?}

Das Medikament ist, so wie es jetzt ist, nachgewiesen wirksam und nachgewiesen sicher. Trotzdem untersuchen wir beide Aspekte auch weiterhin. Das passiert in dreierlei Hinsicht: Zum einen gibt es eine Registerstudie in den USA, die seit der Zulassung durch die FDA läuft. Zum anderen hat die EMA eine sogenannte Post-Authorization Safety Study (PASS) angefordert, die von Novartis im Moment vorbereitet wird. Diese PASS-Studie wird weltweit - außer in 
den USA, dort läuft schon eine Studie - die sicherheitsrelevanten Beobachtungen festhalten. Zusätzlich wird versucht, auch Daten zur Wirksamkeit zu erfassen. Diese Studie wird von mir geleitet und wird zum Ende des Jahres aktiv.

Drittens wird darüber nachgedacht, in Deutschland eine Phase4-Studie zu initiieren, bei der die Wirksamkeit sehr genau untersucht werden soll, auch hinsichtlich klinischer Endpunkte, die in den bisherigen Studien nicht berücksichtigt wurden. So soll noch einmal klarer herausgearbeitet werden, wie die Netzhautfunktion von der Gentherapie profitiert. Es ist z.B. eine sehr wichtige Frage, ob die Therapie nur auf die Stäbchen oder auch auf die Zapfen wirkt.

\section{Was ist mit anderen Gentherapien? Kann man davon ausgehen, dass noch weitere in Zukunft folgen werden?}

Es gibt einige Gentherapien, bei denen man wahrscheinlich davon ausgehen darf, dass sie in den nächsten Jahren ebenfalls zugelassen werden. Da gibt es insbesondere eine sehr weit fortgeschrittene Entwicklung für die Chorioideremie, eine x-chromosomale erbliche Netzhauterkrankung. Da sind wir jetzt sehr weit fortgeschritten in der Phase-III-Studie, an der auch Tübingen als europäisches Referenzzentrum einen großen Anteil hat.

Jetzt wo die erste Gentherapie in der Augenheilkunde zugelassen ist, könnte man ja meinen, dass der Weg für weitere auf jeden Fall schon einmal geebnet ist ...

Ja und nein. Man hat natürlich Erfahrungswerte in der Entwicklung von Gentherapien, die man sich zu Nutze machen kann, um effizienter zu werden. Aber sobald man ein neues Gen in den Vektor schleust und eine andere Krankheit damit therapieren will, dann ist das für die regulatorischen Behörden ein ganz neues Produkt. Und für jedes neue Produkt müssen die wesentlichen Schritte wiederholt werden: Es müssen erneut Phase-I-, Phase-II- und Phase-III-Studien durchgeführt werden, es muss erneut eine Produktion validiert werden etc. Das ist eine große und komplexe Aufgabe, die gerne viele Millionen Euro und viele Jahre braucht. Und ein großes Team an absoluten Experten.

\section{Das klingt nach einem sehr langen Weg.}

Es ist wirklich ein phänomenaler Weg. Ich bin sehr froh, dass ich den von Anfang bis zum Ende mit begleiten darf. In Oxford habe ich ja selbst das Gen kloniert und die viralen Vektoren generiert, habe diese aufgereinigt und die ersten Experimente damit gemacht. Und neulich war ich in Oxford und habe die ersten Patienten gesehen, die mit diesem Vektor behandelt wurden. Es war wirklich ein besonderes Erlebnis, als ich das erste Mal einem 20-jährigen jungen Mann gegenübersaß, der im Wesentlichen das Produkt bekommen hat, das ich damals als PhD-Student entwickelt hatte. Und der mir dann selbst erzählte, dass er auf dem Auge nun deutlich besser sieht. Das ist wirklich ein Gänsehautmoment!

Diese Therapie wird nun weiterentwickelt. Sie ist momentan in der Phase-II-Studie und geht hoffentlich bald in Phase III über. Und hoffentlich zeigt sich, dass wir mit diesem neuen Medikament den Patienten wieder etwas Gutes tun, sodass es zugelassen wird und wir uns dann drüber freuen können, neuen Patienten eine verbesserte Lebensqualität bieten zu können.

\section{Wie lange hat die Entwicklung von Luxturna ungefähr gedauert?}

Das kommt ein bisschen darauf an, wann man den Startpunkt wählt. Man kann den Startpunkt zu dem Zeitpunkt wählen, an dem man herausgefunden hat, welches Gen überhaupt die Erkrankung verursacht. Dann müsste man so in die 90er Jahre zurückgehen.

Man kann da anfangen, wo man sich zuerst überlegt hat, wie man dieses Gentherapieprodukt herstellt, welches Gen man verwendet und wo man es reinsteckt, damit es funktionieren kann.

Oder man könnte auf das Jahr 2000 zurückgehen, wo man das erste Mal bei einem Hund diese Therapie angewandt hat und gesehen hat, dass es funktionieren kann, das wären dann jetzt 19 Jahre. Und die erste Applikation am Menschen war, soweit ich weiß, 2008 oder 2007 - also vor ungefähr 10 Jahren. James Bainbridge führte diese damals in England durch.

Die Entwicklung der Gentherapie fußte auf sehr viel Vorarbeit von so vielen Leuten, dass man sicherlich nicht nur einer Person gratulieren darf. Das zeigt einfach die schönste Seite der akademischen Forschung.

\section{Prof. Dr. Dr. Fischer, haben Sie besten Dank für das Interview!}

\section{Kontaktadresse}

Prof. Dr. Dr. M. Dominik Fischer, FEBO, Department für Augenheilkunde, Universitäts-Augenklinik, Universitätsklinikum Tübingen, Elfriede-AulhornStr. 7, 72076 Tübingen, Deutschland, dominik.fischer@uni-tuebingen.de; Honorary Clinical Research Fellow, Department of Clinical Neurosciences, Nuffield Laboratory of Ophthalmology, University of Oxford, OX3 9DU Oxford, UK. 